\title{
Variations spatiales et temporelles des précipitations des neuf division climatiques de l'oklahoma et implications pour l'utilisation locale de l'indice régional Spatial and temporal variations of precipitation of the nine climate divisions of Oklahoma, and implications for local use of the regional index
}

\author{
F. Rossel et J. Garbrecht
}

Volume 13, numéro 2, 2000

URI : https://id.erudit.org/iderudit/705389ar

DOI : https://doi.org/10.7202/705389ar

Aller au sommaire du numéro

\section{Éditeur(s)}

Université du Québec - INRS-Eau, Terre et Environnement (INRS-ETE)

ISSN

0992-7158 (imprimé)

1718-8598 (numérique)

Découvrir la revue

Citer cet article

Rossel, F. \& Garbrecht, J. (2000). Variations spatiales et temporelles des précipitations des neuf division climatiques de l'Oklahoma et implications pour l'utilisation locale de l'indice régional. Revue des sciences de l'eau / Journal of

Water Science, 13(2), 175-183. https://doi.org/10.7202/705389ar

\section{Résumé de l'article}

L'objectif de cette étude est de quantifier l'importance des variations spatiales des précipitations mensuelles par rapport à leurs variations temporelles régionales à l'échelle des divisions climatiques de l'Oklahoma. Les variations des précipitations à l'intérieur d'une division climatique sont supposées être constituées par trois composantes : les variations spatiales systématiques, les variations temporelles moyennes de la division climatique et les variations aléatoires. Les variations spatiales systématiques sont définies par le gradient des précipitations moyennes sur l'intégralité de la période. Les variations temporelles moyennes de la division climatique sont représentées par les variations temporelles de la moyenne spatiale des précipitations observées aux stations de la division, cette moyenne étant appelée indice régional. Les variations aléatoires sont estimées avec les différences entre les valeurs centrées réduites des précipitations observées aux stations et celles de l'indice régional. Cette étude montre que les amplitudes des variations aléatoires des précipitations mensuelles des neuf divisions climatiques de l'Oklahoma sont significatives par rapport aux variations temporelles régionales. La quantification de l'amplitude des variations aléatoires est importante pour l'utilisation des prévisions régionales des précipitations, car elle permet de déterminer les plages de variation des précipitations locales autour de l'indice régional et donc l'augmentation des risques pris par les utilisateurs des prévisions régionales pour des applications locales. 


\title{
Variations spatiales et temporelles des précipitations des neuf division climatiques de l'Oklahoma et implications pour l'utilisation locale de l'indice régional
}

\author{
Spatial and temporal variations of precipitation \\ of the nine climate divisions of Oklahoma, \\ and implications for local use of the regional index
}

\section{F. ROSSEL ${ }^{*}$, J. GARBRECHT}

Reçu le 5 août 1999, accepté le 7 juin 2000**.

\section{SUMMARY}

\begin{abstract}
The objective of this study is to quantify the magnitude of the spatial variations of the monthly precipitation and relate them to the regional temporal variations of the monthly precipitation at the spatial scale of the climate division of Oklahoma. The precipitation variations within a climate division are assumed to consist of three components: the systematic spatial variations, the mean temporal variations of the climate division, and random variations. The systematic spatial variations are defined with the long-term precipitation gradient. The mean temporal variations of the climate division are represented by the temporal variations of the spatial average of the precipitation observed at the stations included in the climate division; this average is called divisional precipitation. The random variations are estimated with the differences between the standardized values of station and divisional precipitation. This study shows that the magnitude of the random variations of the monthly precipitation of the nine climate divisions of Oklahoma is significant compared to the regional temporal variations. The quantification of the magnitude of the random variations is critical for the use of regional precipitation forecasts, because it allows one to define ranges of local precipitation around the divisional precipitation, and then to quantify the increase of the risk taken by local users of the regional precipitation forecasts.
\end{abstract}

Key-words: monthly precipitation, spatial variation, temporal variation, climate division, divisional value, Oklahoma. 


\section{RÉSUMÉ}

L'objectif de cette étude est de quantifier l'importance des variations spatiales des précipitations mensuelles par rapport à leurs variations temporelles régionales à l'échelle des divisions climatiques de l'Oklahoma. Les variations des précipitations à l'intérieur d'une division climatique sont supposées être constituées par trois composantes : les variations spatiales systématiques, les variations temporelles moyennes de la division climatique et les variations aléatoires. Les variations spatiales systématiques sont définies par le gradient des précipitations moyennes sur l'intégralité de la période. Les variations temporelles moyennes de la division climatique sont représentées par les variations temporelles de la moyenne spatiale des précipitations observées aux stations de la division, cette moyenne étant appelée indice régional. Les variations aléatoires sont estimées avec les différences entre les valeurs centrées réduites des précipitations observées aux stations et celles de l'indice régional. Cette étude montre que les amplitudes des variations aléatoires des précipitations mensuelles des neuf divisions climatiques de l'Oklahoma sont significatives par rapport aux variations temporelles régionales. La quantification de l'amplitude des variations aléatoires est importante pour l'utilisation des prévisions régionales des précipitations, car elle permet de déterminer les plages de variation des précipitations locales autour de l'indice régional et donc l'augmentation des risques pris par les utilisateurs des prévisions régionales pour des applications locales.

Mots clés : pluviométrie mensuelle, variations spatiales, variations temporelles, division climatique, indice régional, Oklahoma.

\section{1 - INTRODUCTION}

Depuis les années 1980 , de nombreux progrès ont été réalisés dans la compréhension des systèmes de circulation atmosphérique globale, des téléconnections entre ces différents systèmes et des interactions entre l'océan et l'atmosphère qui contrôlent les climats continentaux (BARNSTON et al., 1994 ; TRENBERTH et al., 1998). Ces progrès permettent aujourd'hui au Climate Prediction Center de la NOAA (National Oceanic and Atmospheric Administration) de publier des tendances climatiques mensuelles et trimestrielles pour les ÉtatsUnis. Pour que le secteur agricole puisse exploiter pleinement le potentiel offert par les prévisions climatiques, il faut quantifier les relations entre les indices régionaux pour lesquels sont établies les prévisions et les observations locales déterminantes pour l'utilisateur. L'objectif de cet article est de quantifier les variations spatiales des précipitations mensuelles à l'intérieur d'une division climatique et de comparer ces variations spatiales aux variations temporelles des indices régionaux. L'analyse des distributions des différences entre les valeurs centrées réduites des précipitations observées aux stations et celles de l'indice régional doit permettre de déterminer des intervalles de variation des précipitations mensuelles locales autour de l'indice régional. 


\section{2 - DONNÉES}

Le National Climatic Data Center (NCDC) a divisé chaque état des ÉtatsUnis en divisions climatiques (GUTTMAN et QUAYLE, 1995). Les données utilisées sont les séries pluviométriques mensuelles publiées par le NCDC pour les stations des neuf divisions climatiques de l'Oklahoma dont les séries comptent plus de $90 \%$ des 996 valeurs mensuelles des 83 ans de la période 1914-1996. L'Oklahoma, qui fait parti des grandes plaines des États-Unis, présente des paysages de grandes étendues légèrement vallonnées avec une faible pente ascendante vers l'ouest. Le régime saisonnier des précipitations est bimodal avec un maximum principal en mai et un deuxième généralement moins important en septembre. Ce régime est dû aux déplacements de la zone de basse pression qui sépare les masses d'air continental froid et sec en provenance du Canada au nord et les masses d'air maritime chaud et humide provenant du Golfe du Mexique au sud. Les deux périodes de maximum de précipitations, le printemps et l'automne, sont dues à la position moyenne de la zone de basse pression sur l'Oklahoma (JOHNSON et DUCHON, 1995). La réduction des précipitations pendant l'été est due au déplacement de la zone de basses pression vers le nord sur le nord du Kansas et le Nebraska. Les masses d'air maritime chaud et humide, relativement instable, présentes sur l'Oklahoma pendant l'été sont favorables au développement d'orages convectifs qui peuvent être relativement localisés (KESSLER et STADLER, 1993). Les précipitations moyennes hivernales sont inférieures à celles des trois autres saisons, ceci étant dû à la position méridionale de la zone de basses pression sur le centre du Texas. Les précipitations, occasionnellement sous forme de neige, sont fréquemment associées à un front froid, ce qui produit généralement des précipitations de faible intensité mais qui s'étendent sur de grandes étendues dépassant l'échelle des divisions climatiques (LEGATES et DE LIBERTY, 1996).

\section{3 - MÉTHODE}

Les variations des précipitations à l'intérieur d'une division climatique sont supposées être constituées par trois composantes : les variations spatiales systématiques entre les stations de la division climatique, les variations temporelles moyenne de la division climatique et les variations aléatoires. Les variations spatiales systématiques des précipitations à l'intérieur d'une division climatique sont définies par le gradient spatial des précipitations mensuelles moyennes. Le gradient considéré est le gradient moyen sur l'ensemble de la division climatique et a la direction de la plus forte pente ascendante. Les variations spatiales systématiques sont indépendantes du temps. Les variations temporelles moyennes de la division climatique sont représentées par les variations temporelles de la moyenne spatiale des précipitations observées aux stations de la division climatique (ROSSEL et GARBRECHT, 2000). Les variations temporelles moyennes de la division climatique sont indépendantes de l'espace. Cette moyenne spatiale est appelée indice régional. Les variations aléatoires sont estimées avec les différences entre les valeurs centrées réduites 
des précipitations mensuelles de chaque station et celles de l'indice régional (1) ; elles varient dans l'espace et avec le temps. L'utilisation des valeurs centrées réduites permet de supprimer les variations spatiales systématiques. Le calcul de la différence entre les valeurs centrées réduites des stations et celles de l'indice régional permet d'éliminer les variations temporelles moyennes de la division climatique.

$$
\text { Aléa }_{\text {ajt }}=\frac{P_{\text {ajt }}-M_{a j}}{S_{a j}}-\frac{D_{j t}-M_{d j}}{S_{d j}}
$$

où a indique la station, $j$ le mois, $t$ l'année, $P$ est la précipitation mensuelle, $D$ l'indice pluviométrique régional mensuel, $M$ et $S$ les moyennes et les écartstypes sur toute la période d'étude des précipitations mensuelles, I la valeur centrée réduite de l'indice régional, et Aléa la variation aléatoire. Les précipitations mensuelles à un site de la division climatique peuvent donc s'exprimer selon :

\begin{tabular}{|c|c|c|c|c|c|c|}
\hline$P_{\text {ajt }}$ & $=$ & $\mathrm{M}_{\mathrm{aj}}$ & + & $S_{a j} \times I_{j t}$ & + & Saj $\times$ Aléa $_{a j}$ \\
\hline $\begin{array}{l}\text { Pluie } \\
\text { locale }\end{array}$ & $=$ & $\begin{array}{c}\text { Pluie } \\
\text { moyenne }\end{array}$ & + & $\begin{array}{l}\text { Variation } \\
\text { temporelle } \\
\text { régionale }\end{array}$ & + & $\begin{array}{l}\text { Variation } \\
\text { aléatoire }\end{array}$ \\
\hline
\end{tabular}

\section{4 - RÉSULTATS}

Les trois paragraphes suivants sont dédiés à la quantification des trois composantes pour la division centrale de l'Oklahoma et le quatrième aux implications des variations aléatoires pour l'utilisation locale de l'indice régional. Le cinquième paragraphe traite du calcul des variations aléatoires moyennes pour les neuf divisions climatiques de l'Oklahoma.

\subsection{Variations temporelles moyennes de la division climatique}

Les variations inter-annuelles de l'indice régional de la division climatique centrale de l'Oklahoma sont élevées (tableau 1). Elles sont plus importantes par rapport à leur valeur moyenne (coefficient de variation) pendant la période la moins pluvieuse, mais l'amplitude de ces variations (écart-type) est plus importante pendant la saison des pluies.

\subsection{Variations spatiales systématiques à l'intérieur de la division climatique}

Les variations spatiales systématiques de la division climatique centrale de l'Oklahoma sont caractérisées par un gradient croisant relativement important de l'ouest vers l'est avec certaines variations saisonnières (tableau 1). La pente de ce gradient présente deux maximums pendant les périodes les plus humides. Pendant la période estivale, lorsque les précipitations sont fréquemment de caractère orageux et non liées à un front, le gradient est beaucoup 
Tableau 1 Charactéristiques des précipitations mensuelles régionales.

Characteristics of the divisional monthly precipitation.

\begin{tabular}{|c|c|c|c|c|c|c|c|c|c|c|c|c|}
\hline & jan. & fév. & mar. & avr. & mai & juin & juil. & août & sept. & oct. & nov. & déc. \\
\hline Moyenne ${ }^{(1)}$ & 34 & 38 & 62 & 88 & 129 & 109 & 70 & 71 & 96 & 78 & 57 & 40 \\
\hline Écart-Type (2) & 28 & 27 & 39 & 45 & 66 & 58 & 46 & 41 & 60 & 61 & 42 & 32 \\
\hline Coef Variation ${ }^{(3)}$ & 0,82 & 0,71 & 0,63 & 0,51 & 0,51 & 0,53 & 0,66 & 0,58 & 0,63 & 0,78 & 0,74 & 0,80 \\
\hline Direction ${ }^{(4)}$ & NO & NO & NO & ONO & NO & so & so & sSO & ONO & NO & ONO & NO \\
\hline Pente ${ }^{(5)}$ & 10,5 & 11 & 11 & 15,5 & 14,5 & 5 & 9 & 3 & 11 & 14,5 & 10,5 & 11 \\
\hline Pente / éc-typ ${ }^{(6)}$ & $38 \%$ & $41 \%$ & $28 \%$ & $34 \%$ & $22 \%$ & $9 \%$ & $20 \%$ & $7 \%$ & $18 \%$ & $24 \%$ & $25 \%$ & $34 \%$ \\
\hline Moy Diff Abs ${ }^{(7)}$ & 0,30 & 0,30 & 0,33 & 0,40 & 0,40 & 0,44 & 0,44 & 0,48 & 0,40 & 0,30 & 0,28 & 0,27 \\
\hline Éc-typ Diff Abs ${ }^{(8)}$ & 0,31 & 0,31 & 0,28 & 0,35 & 0,34 & 0,40 & 0,40 & 0,42 & 0,33 & 0,32 & 0,28 & 0,26 \\
\hline
\end{tabular}

(1) Moyenne (mm), (2) écarts-types ( $\mathrm{mm}$ ) et (3) coefficient de variation de l'indice pluviométrique mensuel régional de la division climatique centrale de l'Oklahoma, (4) direction et (5) pente $(\mathrm{mm} / 100 \mathrm{~km})$ du gradient pluviométrique, (6) rapport entre la pente et l'écart-type (5/3), (7) moyenne et (8) écarts-types des valeurs absolues des différences entre les valeurs centrées réduites des précipitations observées aux stations et celles de l'indice régional.

(1) Average ( $\mathrm{mm}$ ), (2) standard deviation ( $\mathrm{mm}$ ), and (3) coefficient of variation of the divisional monthly precipitation of the central climate division of Oklahoma, (4) direction and (5) slope $(\mathrm{mm} / 100 \mathrm{~km})$ of the precipitation gradient, (6) ratio between the slope and the standard deviation (5/3), (7) average and (8) standard deviation of the absolute differences between station and divisional standardized values. 
plus faible et presque inexistant en moyenne au mois d'août. La pente du gradient peut-être considérée comme l'amplitude moyenne des variations spatiales systématiques, elle varie de 7 à $41 \%$ des écarts-types mensuels de l'indice régional entre août et février.

\subsection{Variations aléatoires à l'intérieur de la division climatique}

Les différences entre les valeurs centrées réduites des précipitations mensuelles des stations et celles de l'indice régional obtenues pour la division climatique centrale de l'Oklahoma montrent que les précipitations observées aux stations et l'indice régional peuvent avoir des différences relativement importantes. Même si la majorité des différences sont inférieures à $( \pm)$ un demi-écarttype, $25 \%$ des différences sont supérieures à $( \pm)$ un demi-écart-type, $6 \%$ sont supérieure à $( \pm)$ un écart-type et les valeurs extrêmes sont proches de trois écarts-types. La distribution de ces différences est voisine d'une distribution normale. La moyenne des valeurs absolues des différences varie entre 0,27 écartstypes en décembre et 0,48 en août avec un maximum pendant la période estivale lorsque les précipitations sont de caractère orageux (tableau 1). La moyenne des douze moyennes mensuelles est de 0,36 écarts-types pour la division climatique centrale de l'Oklahoma.

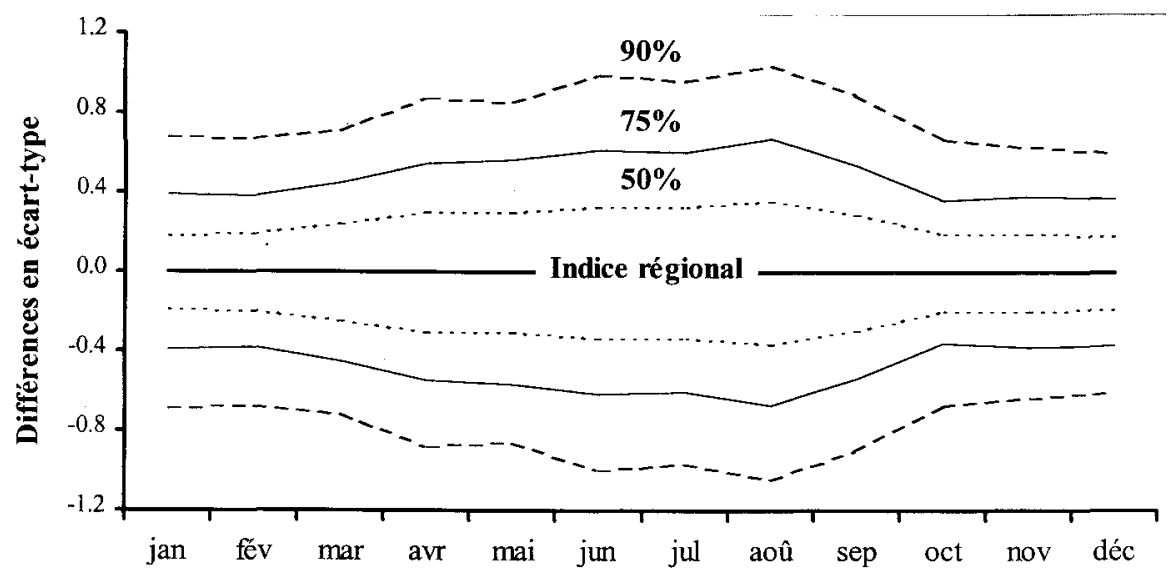

Figure 1 Intervalles autour de l'indice régional à l'intérieur desquels 50, 75 et $90 \%$ des différences entre les valeurs centrées réduites des précipitations observées aux stations et celles de l'indice régional ont été observées.

Intervals around the divisional value within which 50, 75 and $90 \%$ of the differences between stations and divisional precipitation values have been observed.

\subsection{Implications pour l'utilisation locale de l'indice régional}

L'étude des distributions des différences entre les valeurs centrées réduites des séries des stations et de l'indice régional permet de définir des intervalles de variation autour des valeurs de l'indice régional (figure 1). Ces intervalles 
constituent des « enveloppes " à l'intérieur desquelles 50,75 et $90 \%$ des précipitations mensuelles locales ont été observées. Pour la division climatique centrale de l'Oklahoma, ces « enveloppes " sont plus larges d'avril à septembre avec des valeurs proches d'un écart-type pour l'enveloppe $90 \%$ de juin à aôut. La probabilité d'observer localement des précipitations proches de l'indice régional est donc plus élevée pendant la période hivernale. En d'autres termes, indépendamment de la fiabilité des prévisions, l'augmentation du " risque » dû à l'utilisation d'un pronostic régional pour la gestion locale est moins grande l'hiver que l'été. On peut obtenir l'intervalle dans lequel la probabilité d'observer les précipitations locales est égale à $50 \%$ à partir de l'équation (2) selon :

$$
P_{50 \% a j t}=M_{a j}+S_{a j} \times I_{j t} \pm S_{a j} \times L_{50 \% j}
$$

où $L_{50 \%}$ est la limite de l'enveloppe $50 \%$. Si l'indice régional est issue d'une prévision, l'expression (4) devient:

$$
P_{50 \% a j t}=M_{a j}+S_{a j} \times\left(l_{j t} \pm E_{j t}\right) \pm S_{a j} \times L_{50 \% j}
$$

où $E$ est l'erreur associée à la prévision.

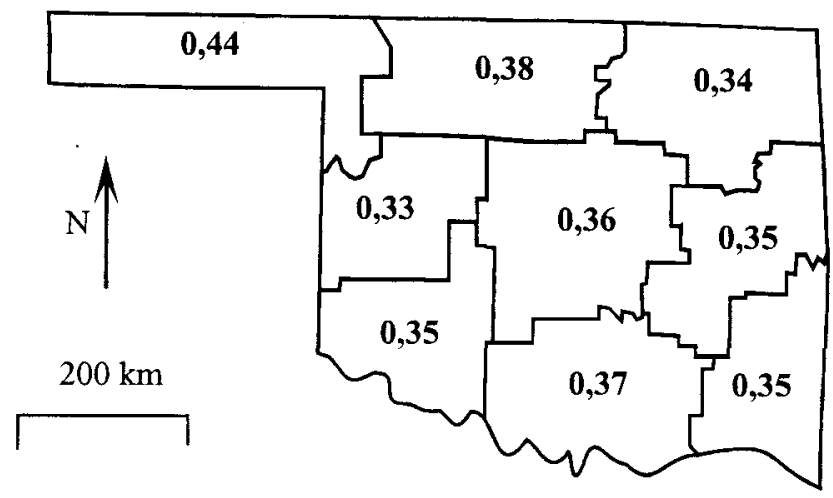

Figure 2 Moyennes annuelles des valeurs absolues des différences entre les valeurs centrées réduites des précipitations mensuelles observées aux stations et celles des indices régionaux des neuf divisions climatiques de l'Oklahoma.

Yearly average of the absolute differences between the standardized values of monthly stations and divisional precipitation values for the nine climate divisions of Oklahoma.

\subsection{Extension à l'ensemble de l'état de l'Oklahoma}

Les moyennes mensuelles des valeurs absolues des différences entre les valeurs centrées réduites des précipitations mensuelles de chaque station et celles de l'indice régional des neuf divisions climatiques de l'Oklahoma sont du même ordre de grandeur. Elles ont des variations saisonnières comparables avec un maximum d'avril à septembre et un minimum d'octobre à mars. Seules les valeurs de la division climatique située au nord-ouest sont légèrement plus 
importantes ; ceci est essentiellement lié à la plus grande extension géographique de la division climatique, $400 \mathrm{~km}$ de long contre 200 à $300 \mathrm{~km}$ pour les huit autres (figure 2). Les mêmes précautions devront donc être prises pour l'utilisation locale de l'information régionale pour les neuf divisions climatiques de l'Oklahoma.

\section{5 - CONCLUSION}

L'objectif de cette analyse était de quantifier et de comparer les variations spatiales et temporelles des précipitations mensuelles d'une division climatique de l'Oklahoma. La quantification des différences entre les observations locales et l'indice régional devait permettre de définir des plages d'incertitudes pour l'utilisation locale des prévisions régionales. Les variations temporelles des précipitations mensuelles de la division climatique centrale de l'Oklahoma sont élevées. Les écarts-types sur la période 1914-1996 sont compris entre $27 \mathrm{~mm}$ en février et $66 \mathrm{~mm}$ en mai pour des précipitations moyennes comprises entre $34 \mathrm{~mm}$ en janvier et $129 \mathrm{~mm}$ en mai. Les variations spatiales systématiques peuvent être équivalente à $40 \%$ des écarts-types pendant la période hivernale. Elles sont en revanche beaucoup plus faibles pendant l'été avec un minimum de $7 \%$ en août. Les variations aléatoires sont plus importantes encore, elles sont comprises entre $27 \%$ des écarts-types en décembre et $48 \%$ en août. Elles sont maximum l'été lorsque les précipitations sont fréquemment de caractère orageux et minimum l'hiver lorsque les précipitations sont fréquemment de grandes étendues associées au passage de fronts froids. Les variations aléatoires des neuf divisions climatiques de l'Oklahoma ont les mêmes variations saisonnières et sont du même ordre de grandeur avec des moyennes proches de 0,36 écarts-types.

Cette étude montre que les variations spatiales systématiques et aléatoires des précipitations mensuelles des divisions climatiques de l'Oklahoma peuvent être équivalente à plus de $40 \%$ des variations temporelles. Donc, si un indice régional pour l'ensemble d'une division climatique est représentatif des variations temporelles globales des précipitations, les écarts à la normale observés aux stations peuvent avoir des différences significatives avec ceux de l'indice régional. Il est important pour le transfert de l'échelle régionale à l'échelle locale de noter que les variations spatiales systématiques peuvent être estimées alors que les variations spatiales aléatoires sont imprévisibles. Cette étude a permis de définir des plages de variation des précipitations locales autour de l'indice régional. La quantification des différences entre stations et indice régional est une étape indispensable pour l'utilisation locale des prévisions régionales. 


\section{RÉFÉRENCES BIBLIOGRAPHIQUES}

BARNSTON A., VAN DEN DOOL $\mathrm{H}_{\text {, }}$ ZEBIAK S., BARNETT T., JI M. RODENHUIS D., CANE M., LEETMAA A., GRAHAM N., ROPELEWSKI C., KOUSKY V., O'LENIC E., LIVEZEY R., 1994. Longlead seasonal forecast : where do we stand ? Bull. Am. Met. Soc., 75, 20972113.

GUTTMAN N., QUAYLE R., 1995. A historical perspective of U.S. climate divisions. NCDC, Asheville, NC, USA.

JOHNSON H.L., DUCHON C.E., 1995. The atlas of Oklahoma climate. University of Oklahoma Press, Norman, OK, USA.

KESSLER E., STADLER S. 1993. Some warm-season singularities in central Oklahoma precipitation. Oklahoma Climatological Survey, Norman, OK, USA, Technical report $n^{\circ}$ 93-001, $55 \mathrm{p}$.
LEGATES D., DELIBERTY T., 1996. Precipitation in the southern Great Plains : observations and model simulations of present-day and doubled atmospheric $\mathrm{CO}_{2}$ concentrations. Global Climate Change Response Program, USDI, Denver, CO, USA.

ROSSEL F. GARBRECHT J., 2000. Analyse et amélioration d'un indice pluviométrique mensuel régional pour les grandes plaines du sud des États-Unis. Rev. Sci. Eau, 13, 1, 39-46.

TRENBERTH, K.E., BRANSTATOR G.W., KAROLY D., KUMAR A., LAU N.C., ROPELEWSKI C., 1998. Progress during TOGA in understanding and modeling global teleconnections associated with tropical sea surface temperature. J. Geophys. Res., 103, 14291-14324. 